\title{
Psychological Sequelae of Road Traffic Accidents: An Inadequately Addressed Problem
}

\author{
ALAIN DI GALLO and WLLLAMLI. PARRY-JONES
}

In 1994, 3650 people died on the roads in the UK and 46531 were injured seriously. Altogether, 315189 road accident casualties, of various degrees of severity, were recorded. Other European countries report similar, or even worse, statistics of road deaths (Department of Transport, 1995). Paradoxically, the road traffic environment is not regarded as a major traumatising force. Unlike air or train crashes, road traffic accidents have become an integral part of daily experience. They are dispersed in their nature, small in size, but huge in totality. When a community is struck by the loss of several of its members in a bus crash, the psychological impact on survivors is likely to be recognised publicly, but, generally, the consequences of individual road traffic accidents attract little attention. The fact that they can generate exceptional stress for the victims and a wide range of psychological disturbances has been corroborated in recent years. Among the increasing number of investigations about the psychological impact of road traffic accidents, however, there appears to have been only one systematic, prospective study. Mayou et al (1993) assessed 188 consecutive adult victims with multiple injuries, or with whiplash neck injury, following a car or motorcycle accident. Eighteen per cent showed acute distress, characterised by anxiety or depression, in combination with "horrific" intrusive memories of the accident. Twelve months after the accident, almost a quarter of the subjects described psychiatric problems of three strongly related types, namely mood disorder (depression, anxiety), post-traumatic stress disorder (PTSD) and travel phobia. The occurrence of acute distress was associated with poor psychological outcome at one year. A number of other studies, investigating less representative samples, or concerned exclusively with patients referred for psychological help or assessment following road traffic accidents, generally reported higher rates of psychiatric sequelae. Despite the evidence that adverse reactions are common, however, several relevant aspects of psychological morbidity following road traffic accidents have not yet been addressed adequately. Some of these will be discussed and future research directions outlined.

\section{Incidence and range of pyychological sequelae}

The frequency of post-accident psychological disturbances remains controversial. Different studies investigate different types of accidents (e.g. car crashes or pedestrian accidents) and use more or less randomly selected samples and different assessment instruments, frequently incorporating new interview schedules and self-report questionnaires. Even if the same instrument is used, the results may be influenced by its differential use as a self-report questionnaire or as an interview schedule. It is often difficult, therefore, to compare studies. This suggests that the refinement of the available measuring tools should be a priority of further research.

\section{Significance of the severity of accidents on psychological outcome}

While it might be predicted that severe road traffic accidents would produce seriously adverse reactions, there is evidence that minor crashes can also evoke marked stress. However, the precise nature of the relationship between the range of psychological sequelae and the severity of the accident has not been established. In an investigation of patients referred for psychiatric consultation following automobile accidents (Goldberg \& Gara, 1990), depression was found to be three times more frequent than PTSD. The authors concluded that the accident might have been capable of triggering depression in certain patients, but was not "traumatic" enough to induce PTSD. In contrast, Mayou et al (1993) emphasised the need to be aware of all the psychological consequences for those victims who suffered only minor injury, 
together with those who were uninjured. PTSD, mood disorders and travel phobia occurred as frequently in the modestly-injured "whiplash group", as in patients with multiple injuries. The principal predictor for PTSD was the rating of "horrific" memories and PTSD did not occur in individuals who had amnesia for the accident. It follows that the consequences of an outwardly "trivial" accident, that attracts little or no medical attention, should never be overlooked. Such oversight may occur easily because accident victims tend to avoid memories of the traumatic experience or may fear being derided if they admit to psychological problems.

\section{Pre-accident disposition and vulnerability}

Although, in many cases, a close link between cause (accident) and effect (psychological sequelae) is apparent, adverse reactions do not always occur. This raises the question why some individuals seem to be resistant to post-accident disturbance and whether, in other cases, the accident simply operates as a trigger to activate pre-existing mental disorders. The assessment of the pre-accident disposition of the patient, which necessarily has to be undertaken retrospectively, often proves to be problematic. Under the immediate stress of the accident, victims tend to deny or minimise preexisting problems, since they may seem irrelevant to the actual situation. A range of complex forensic issues stems from the consequences of road traffic accidents. In compensation litigation, the question of vulnerability is often crucial and there may be a risk of the lawyer over-using the diagnosis of PTSD in favour of the plaintiff. PTSD provides a more obvious and understandable link between external causation and effect, as opposed to a possible assumption of internal causation, as might be the case in depression, anxiety, or adjustment disorder (Platt \& Husband, 1986). However, PTSD cannot be excluded from the discussion of vulnerability. Platt \& Husband (1986) hypothesised that people who developed PTSD following an automobile accident of moderate severity were likely to be individuals whose "reserve capacity" to cope successfully with the exigencies of daily life was low. Blanchard et al (1995) showed that previous major depression, or depression at the time of a motor vehicle accident, predisposed to the development of PTSD. Individual differences in adverse psychological responses to road traffic accidents and the factors that influence vulnerability to developing post-traumatic stress reactions clearly warrant further investigation. The variability in individual cognitive appraisal of the trauma, the coping efforts available, and the reactions of the social environment are factors of particular importance. Greater experience and evidencebased expertise in this field would facilitate the early identification of individuals who are at risk.

\section{Treatment}

Accounts of the treatment of individuals suffering from psychological problems following road traffic accidents are based mainly on case reports (Briggs, 1993; Spector \& Huthwaite, 1993) or on general experience with post-traumatic stress reactions. There have been no adequately controlled studies and the value of medication and psychotherapy still lack comprehensive investigation. The literature indicates that acute psychological reactions to a road traffic accident often are a reliable predictor of long-term disturbances. Early intervention, therefore, may prevent chronic psychopathology, but the benefits have to be demonstrated by rigorous evaluation studies, in order to justify the costs and therapeutic effort involved.

\section{Psychological sequelae in children and adolescents}

Children are among the most vulnerable road users. Roads are designed by adults for adults and the ability of a child to cope with the demands of a traffic-ridden environment must always be considered in relation to age and developmental stage. Road traffic accidents account for approximately two-thirds of accidental deaths and for a quarter of all deaths among schoolchildren (Department of Health, 1991). In 1994, in the UK, more than $50 \%$ of pedestrians severely injured on the roads were children aged under 16 years. Very little is known about their psychological responses, the natural history of their disorders or the predictors of outcome. There is still a lack of systematic research into the range and management of the psychological sequelae of road traffic accidents in children and young people. Publications consist mainly of case reports (Jones \& Peterson, 1993). The findings of the authors' exploratory investigation indicated that about $15 \%$ of the children and adolescents assessed suffered from PTSD or serious trafficrelated fears three months after a road traffic accident. Parents reported increased mood disturbance in their children compared with the preaccident period. High levels of distress during, and 
immediately after, the accident were associated with severe post-traumatic stress symptoms.

\section{Conclusions}

The knowledge of psychological sequelae following road traffic accidents is less than comprehensive and there is an unequivocal need for further research. The present strong evidence that disturbances are common, carries immediate implications for all health care staff engaged in the management of road traffic accident victims, particularly those working in hospital casualty departments and in general practice. It is imperative that staff training should include information about the potential psychological stress reactions following accidental trauma and about risk factors, such as high levels of fear of death experienced during the accident. Well-intentioned remarks, like "you have been lucky, you could be much worse off", can do more harm than good. Excellent information leaflets published for distribution by hospitals and medical practices are available (e.g. Child Accident Prevention Trust, 1994). When general practitioners see patients for post-accident checks, they should explain to them the range of possible psychological sequelae of road traffic accidents and enquire about any relevant symptoms. Unfortunately, the notion that mentioning and discussing symptoms causes them to occur, still impedes their open acknowledgement.

\section{Acknowledgements}

This work was supported by the Swiss National Science Foundation, the Freiwillige Akademische Gesellschaft (Basel), and the Janggen-Poehn-Stiftung (St. Gallen).

\section{References}

Blanchard, E. B., HicxlnNo, E. J., TAYLor, A. E., el al (1995) Psychiatric morbidity associated with motor vehicle accidents. Journal of Nervous and Mental Disease, 183, 495-504.

Briggs A. C. (1993) A case of delayed post-traumatic stress disorder with organic 'memories' accompanying therapy. British Journal of Psychiatry, 163, 828-830.

Chimd Accident Prevention Trust (1994) Getting Over an Accident. How to Help your Child. London: CAPT.

Department of Healtr (1991) The Health of the Nation. London: HMSO.

Department of Transport (1995) Transport Statistics Great Britain. London: HMSO.

GolDBERG, L. \& GARA, M. A. (1990) A typology of peychiatric reactions to motor vehicle accidents. Psychopathology, 23, 15-20.

Jones, R. W. \& Peterson, L. W. (1993) Posttraumatic stress disorder in a child following an automobile accident. Journal of Family Practice, 36, 223-225.

Mayou, R., Bryant, B. \& Dutrie, R. (1993) Psychiatric consequences of road traffic accidents. British Medical Journal, 307, 647-651.

Platt, J. J. \& HusandD, S. D. (1986) Posttraumatic streas disorder in forensic practice. American Journal of Forensic Psychology, 4, $29-95$.

SPECTOR, J. \& HUTHWATrE, M. (1993) Eye-movement desensitisation to overcome post-traumatic stress disorder. British Journal of Psychiatry, 163, 106-108.

Alain Di Gallo, MD, William Ll. Parry-Jones, FRCPsych, Department of Child and Adolescent Psychiatry, University of Glasgow, Scotland

Correspondence: Dr Alain Di Gallo, Department of Child and Adolescent Psychiatry, University of Glasgow, Caledonia House, Royal Hospital for Sick Children, Yorkhill, Glasgow G3 8SJ

(First received 11 December 1995, final revision 2 April 1996, accepted 18 April 1996) 